\title{
Comparative Study of Tribological Behaviours of Different Base Greases Enhanced by Graphene Nano Platelets
}

\author{
Jankhan Patel, Amirkianoosh Kiani \\ Silicon Hall: Laser Micro/Nano Fabrication Facility, Faculty of Engineering and Applied Science, Ontario \\ Tech. University (UOIT) \\ 2000, Simcoe St. north, Oshawa, Canada \\ Jankhankumarnayankumar.patel@uoit.net; Amirkianoosh.kiani@uoit.net
}

\begin{abstract}
Graphene is one of the strongest allotropes in carbon family. Applications of graphene are found in many industries such as coating, sensors, electronics, light processing, energy, and environmental sectors. Myriad studies have proven that graphene has excellent tribological capabilities. In fact, multilayer structure of graphene allows layers to shear between the mating surfaces to reduce friction. In addition, its extensive mechanical properties allow graphene particles to work as nano bearings to mitigate metal-to-metal contact and reduce wear. In this study, graphene nano particles were evaluated at $1 \% \mathrm{w} / \mathrm{w}$ concentration using the lithium-base general purpose grease (NL-1), the water proof general purpose grease (NL-2) and the extreme pressure grease (NL-3). To characterize graphitic defects and topography of graphene platelets, micro-Raman spectroscopy and transmission electron microscopy (TEM) were utilized at high magnification. For tribological evaluation, shaft-on-plate tribometer was used to test grease at different loads and rotating speeds. The results show that all three nano greases have lower average friction coefficient (AFC) in comparison with control sample (pure grease). Among them, the water resistive grease (NL-2) has the best performance followed by the extreme pressure grease (NL-3) and the lithium based grease (NL-1) respectively.
\end{abstract}

Keywords: Water resistant grease, Lithium grease, White grease, Graphene nano platelets.

\section{Introduction}

Friction is very essential in daily life activities; however, it also damages the performance of machine and cause heat generation on the moving surfaces. Higher concentration of frictional heat can alter the material properties of the surface and initiate wear of the substrate. Increment in wear can greatly affect the sustainability and damage overall performance of the machines. To enhance the friction and wear resistivity of machine components, wide range of lubricants are deployed between the mating surfaces. Myriad studies have been conducted on different base lubricants to improve contact surface interaction. From all those studies, applications of nano particles have drastically shifted lubrication industries.

Liquid, semi-liquid and solid lubricants are applied for diverse applications based on the requirements. Liquid lubricants are utilized to maintain cleanliness and remove heat concentration due to friction. Conversely, grease is found as semi-liquid lubricant, which is mainly applied when substrate requires constant contact with lubricants. In addition, sticky nature of grease provides sustained hydrodynamic regime between the contact surfaces. Solid lubricants are deployed under high load and temperature conditions. Along with that, solid lubricants are highly efficient when contact surfaces are exposed to environment which can destroy liquid and semi-liquid lubricants. Many studies have been reported to see effect of different nano additives in to all three forms of lubricants [1-2]. Out of different nano additives, carbon based nano additives have drawn attention of researchers due to its extensive physical, chemical properties and easy of fabrication. Different forms of carbon additives were evaluated in liquid lubricant at various concentrations and shown great reduction in friction and wear without affecting physical and chemical properties of lubricants [3-5]. In case of semi liquid lubricants, different forms of carbon additives has disclosed modification of friction and wear.

Greases are classified according to the base lubricant, additive and thickeners. In this study three different base greases were evaluated at $1.0 \% \mathrm{w} / \mathrm{w}$ concentration of graphene nanoplatelets and compared with respect to frictional resistivity. The water proof general purpose grease has anhydrous calcium as a thickener and application of this grease is mainly in cars, public machines and agriculture industry. However, lithium grease contains lithium as a thickener and zinc was added to 
maintain white colour to identify life of grease based upon change in colour. Finally, extreme pressure grease are applied in rolling, mining, steel industries where bearing are deployed to support higher load and vibrations.

\section{Experimental setup}

Graphene nano platelets were manufactured using modified hummers method in single sealed jar [6]. Using hydrazine monohydrate, graphene nanoplatelets were obtained from graphene oxide. The nano greases were prepared at Kennedy Labs using three base greases: the lithium base general purpose grease, the water resistant grease and the extreme pressure grease. Graphene nano platelets were measured using extremely precise weight scale and added to all three grease at $1.0 \% \mathrm{w} / \mathrm{w}$ concentration. The nano grease samples were characterized as lithium base nano grease - NL1, water based nano grease - NL2 and extreme pressure grease - NL3. For better dispersibility of nano additives in the grease, direct mixing method was applied [7]. Using mechanical mixture grease and nano additives were rigorously amalgamated for 30 minutes.

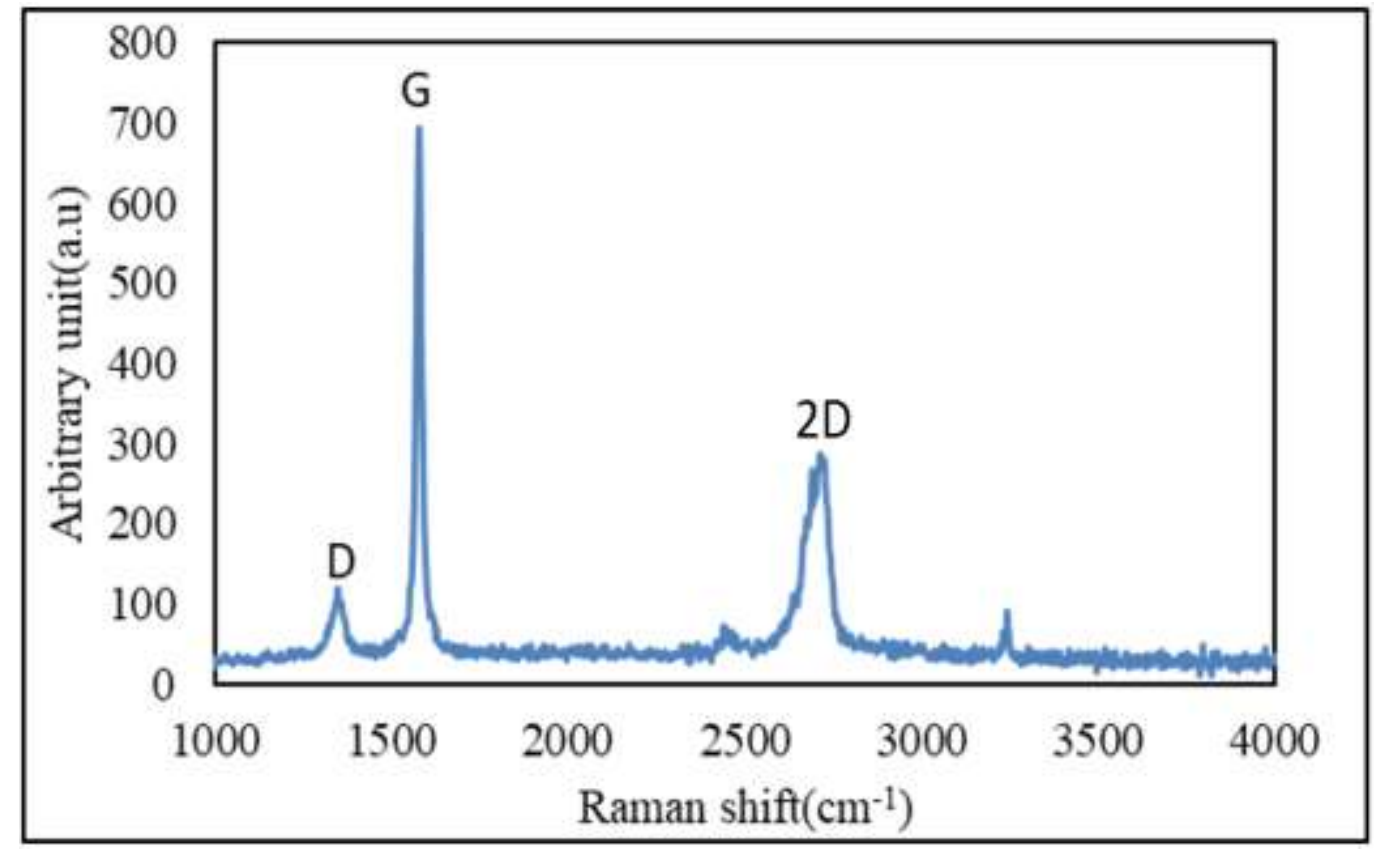

Fig. 1: Raman spectroscopy of graphene Nano platelets.

Raman characterization was performed at $514 \mathrm{~nm}$ laser frequency. The raman spectra as shown in Fig.1 reveals D band at $\sim 1351 \mathrm{~cm}^{-1}$, G band at $\sim 1581 \mathrm{~cm}^{-1}$ (G band), and 2D band was stretched at $\sim 2713 \mathrm{~cm}^{-1}$. The intensity of D band demonstrate defects in carbon lattice where $G$ band discloses $E_{2 \mathrm{~g}}$ phonons, which leads to the stretching in $\mathrm{sp}^{2}$ carbon pair in rings or chains. As shown in Fig.1, narrow 2D band revels less disorder in carbon lattice. Adding to that intensity ratio was calculated for $D$ band and $G$ band as $\left(I_{D} / I_{G}\right)$ as 0.17 . Comparatively, lower intensity ratio shows less defected graphene nano platelets.

As shown in Fig. 2, TEM images were obtained at two different scales to evaluate dense structure of nano particles and the nature of graphene layers. Fig.2 (A) disclose that graphene particles are not highly aggregated but found widely scattered in the epoxy mixture. Due to graphene conglomeration issue, graphene particles were mixed with epoxy and sliced in to nano sheets. The magnified image in Fig.2 (B) revels multilayers of graphene classified based upon variation in colour. Graphene thin nano platelets were in transparent and deposited like crumpled silk. Adding to that, no wrinkles were investigated on nano platelets morphology. Fig.2 (C) manifests three nano greases vary in colour after mixing with graphene nano particles. 

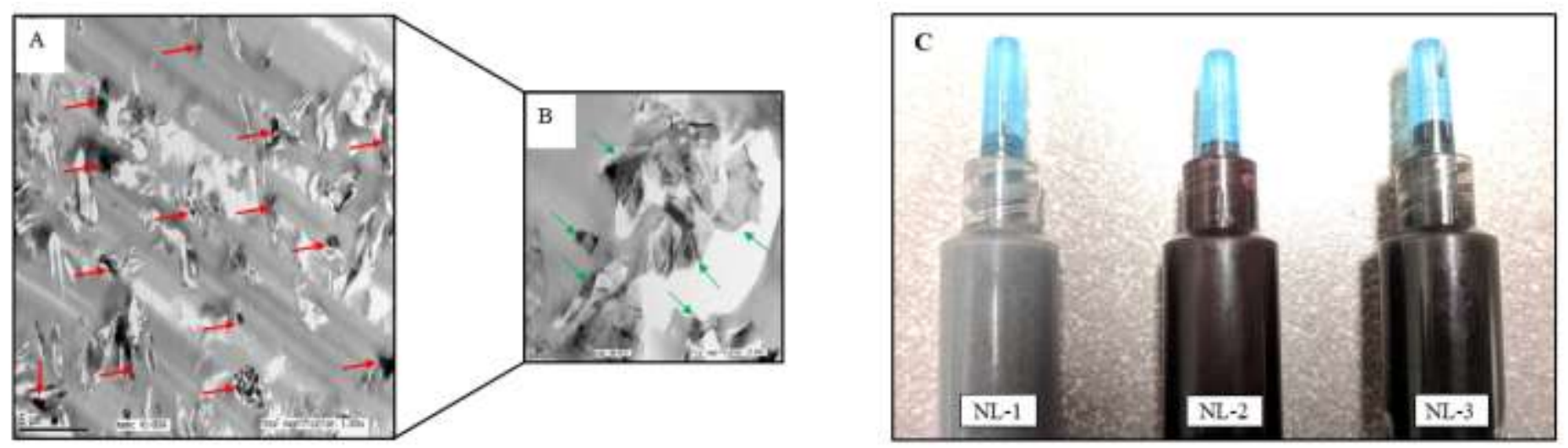

Fig. 2: TEM images were shown in A and B at different magnifications (red arrow demonstrated graphene nano platelets, green arrow characterize multilayers of graphene based upon variation in colours.); Three nano grease samples were demonstrated in Figure: $\mathrm{C}$

(TEM scale for $\mathrm{A}$ is $5 \mu \mathrm{m}$ and for $\mathrm{B}$ is $1 \mu \mathrm{m}$ ).

\section{Experimental results}

The friction coefficient test was conducted using shaft-on-plate tribometer. To analyse the effect of nano greases at different loading conditions, all greases were tested at $20 \mathrm{~N}, 25 \mathrm{~N}$ and $30 \mathrm{~N}$ loading conditions. The tangential load was applied on the shaft using two parallel plate and variation in friction coefficient was calculated. In addition, each test was performed at 120 RPM for 5 min intervals. After performing each test specimen were ultrasonicated and cleaned using ethanol as per the operating instruction. As shown in Fig.3 graphene enhanced lithium grease (NL-1) has comparatively higher friction coefficient for all three loading conditions in comparison with NL-2 and NL-3; but it is considerably lower than control sample (PG). However, the average friction coefficient (AFC) of graphene enhanced extreme pressure nano additive grease (NL-3) is found in between the other two nano greases. Also, the AFC for NL-3 is lower than control sample (PG). Moreover, graphene enhance water resistive grease (NL-2) demonstrated the least friction coefficient compare to all other greases including control sample.

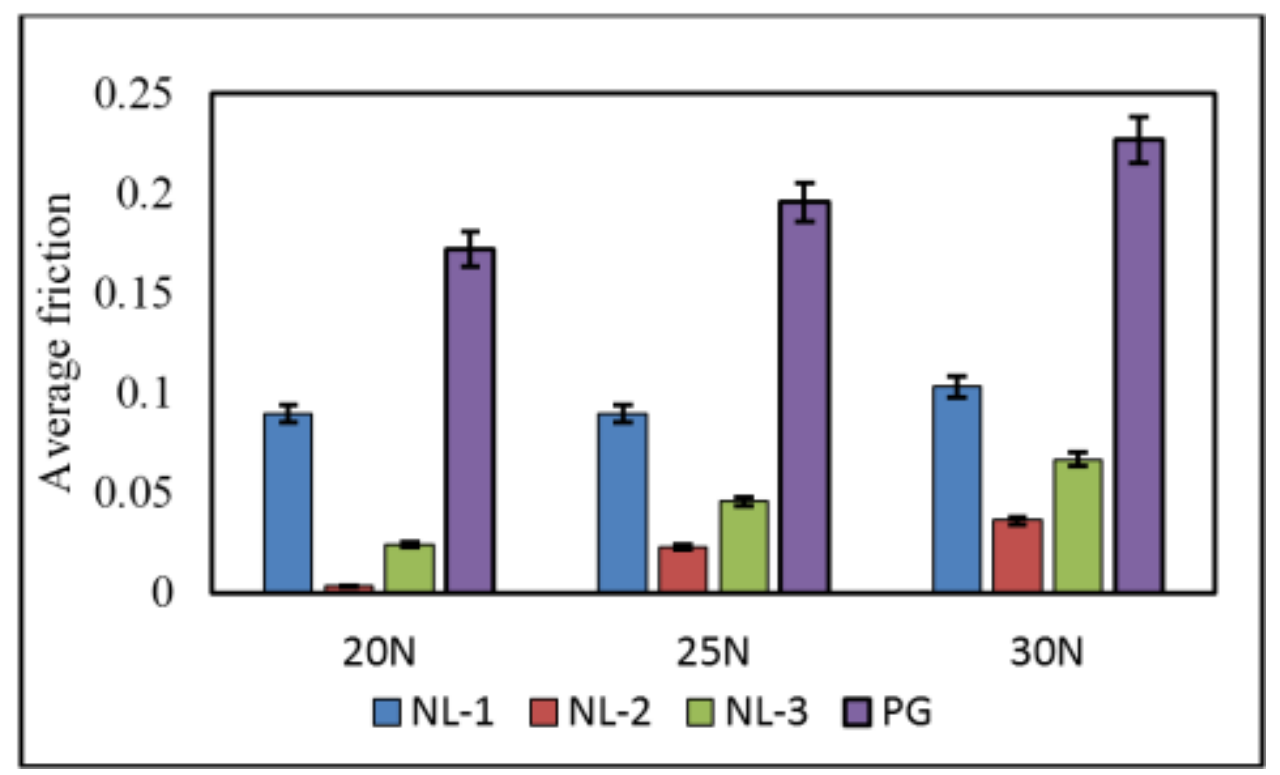

Fig. 3: Friction coefficient results of three nano grease at 3 loading conditions. 


\section{Conclusion}

In this study, graphene was manufactured using modified hummers method in a single sealed jar. Material topography and Raman characterization reveals that multilayer graphene has low defect in lattice structure, which does not allow oxygen particles to deposit between the layers. TEM morphology reveals that graphene platelets are not highly aggregated which will allow particles to disperse homogenously in the grease. Frictional coefficient test results demonstrate that the lithium grease (NL-1) has highest friction coefficient at all three loading conditions of 20, 25 and 30N. The water resistant grease (NL-2) showed lowest friction coefficient and the extreme pressure grease (NL-3) has comparatively lower AFC than the lithium grease but higher than the water-resistant grease. The findings in this research can lead to promising solutions for synthesis of enhanced nano greases with tailored tribological behaviours for wide range of applications.

\section{Acknowledgements}

The author would like to acknowledge the NSERC for funding this study and Dr. Ahmed Barari for his support for rotatory plate-on-shaft friction system.

\section{References}

[1] H.-J. Song, N. Li, "Frictional behavior of oxide graphene nanosheets as water-base lubricantadditive," Applied Physics A., vol. 105 , no. 4 , pp. 827-832, 2011.

[2] K. Lee, Y. Hwang, S. Cheong, Y. Choi, L. Kwon, J. Lee, S. H.Kim, "Understanding the role of nanoparticles in nanooil lubrication," Tribology Letters, vol. 35, no. 2, pp. 127-131, 2009.

[3] J. Patel, A. Kiani, "Effects of reduced graphene oxide (rgo) at different concentrations ontribological properties of liquid base lubricants," Lubricants, vol. 7, no. 2, pp. 1-11, 2019.

[4] B. M. Kamel, A. Mohamed, M. El Sherbiny, K. Abed, M. Abd-Rabou, "Tribological proper-ties of graphene nanosheets as an additive in calcium grease," Journal of Dispersion Science and Technology, vol. 38, no. 10, pp. 1495-1500, 2017.

[5] C.-G. Lee, Y.-J. Hwang, Y.-M. Choi, J.-K. Lee, C. Choi, J.-M. Oh, "A study on the tribologi-cal characteristics of graphite nano lubricants," International Journal of Precision Engineering and Manufacturing, vol. 10, no. 1, pp. 85-90, 2009.

[6] L. Shahriary, A. A. Athawale, "Graphene oxide synthesized by using modified hummersapproach," Int. J. Renew. Energy Environ. Eng, vol. 2, no. 1, pp. 58-63, 2014.

[7] S. R. Nam, C. W. Jung, C.-H. Choi, and Y. T. Kang, "Cooling performance enhancement of LED (light emitting diode) packages with carbon nanogrease," Energy, vol. 60, pp. 195-203, 2013. 